CERN-TH.6609/92

ILL-(TH)-92\# 16

\title{
SPECTROSCOPY, EQUATION OF STATE AND MONOPOLE PERCOLATION IN LATTICE QED WITH TWO FLAVORS
}

\author{
S. J. HANDS, A. KOCIĆ \\ Theory Division, CERN, CH-1211 Geneva 23, Switzerland \\ J. B. KOGUT \\ Department of Physics, University of Illinois, Urbana, IL 61801-3080, USA \\ R. L. RENKEN \\ Physics Department, University of Central Florida, Orlando, FL 32816, USA \\ D. K. SINCLAIR \\ High Energy Physics Division, Argonne National Laboratory, Argonne, IL 60439, USA \\ K. C. WANG \\ Australian National University, Canberra, A.C.T. 2600 Australia
}

\begin{abstract}
Non-compact lattice QED with two flavors of light dynamical quarks is simulated on $16^{4}$ lattices, and the chiral condensate, monopole density and susceptibility and the meson masses are measured. Data from relatively high statistics runs at relatively small bare fermion masses of $0.005,0.01,0.02$ and 0.03 (lattice units) are presented. Three independent methods of data analysis indicate that the critical point occurs at $\beta=0.225(5)$ and that the monopole condensation and chiral symmetry breaking transitions are coincident. The monopole condensation data satisfies finite size scaling hypotheses with critical indices compatible with four dimensional percolation. The best chiral equation of state fit produces critical exponents $\left(\delta=2.31, \beta_{m a g}=0.763\right)$ which deviate significantly from mean field expectations. Data for the ratio of the sigma to pion masses produces an estimate of the critical index $\delta$ in good agreement with chiral condensate measurements. In the strong coupling phase the ratio of the meson masses are $M_{\sigma}^{2} / M_{\rho}^{2} \approx 0.35$, $M_{A_{1}}^{2} / M_{\rho}^{2} \approx 1.4$ and $M_{\pi}^{2} / M_{\rho}^{2} \approx 0.0$, while on the weak coupling side of the transition $M_{\pi}^{2} / M_{\rho}^{2} \approx 1.0$, $M_{A_{1}}^{2} / M_{\rho}^{2} \approx 1.0$, indicating the restoration of chiral symmetry.
\end{abstract}

CERN-TH.6609/92

August 1992 


\section{Introduction and Review}

This paper continues and sharpens our series of studies of noncompact QED incorporating two species of light dynamical fermions. The goal of this paper will be to establish that monopole condensation in this theory is a legitimate phase transition with power-law critical singularities, and that this transition is coincident with chiral symmetry breaking. The critical properties of the chiral symmetry breaking transition will be studied using the theory's meson spectrum and chiral condensate. New methods of analyzing the simulation data of the light quark sector of the theory will suggest, as proposed in past work, that the critical point is characterized by a non-trivial, interacting field theory. Although this paper will emphasize new simulation results and will leave parallel theoretical developments to other articles, we begin with some general remarks and perspectives. One of our motivations for this set of studies is to understand whether realistic strongly coupled field theories exist in four dimensions, and if so, to extract their essential ingredients. In the language of the renormalization groups, we are searching for ultra-violet stable fixed points which are non-trivial. We accept the conventional wisdom that $\lambda \phi^{4}$ is trivial in four dimensions and turn to theories with gauge fields. QED is the simplest candidate in this direction and if we let its charged fields be fermionic, chiral symmetry can protect the model from unnatural fine-tuning problems. An early excursion in this field found that the compact lattice version of QED coupled to dynamical fermions had a first order chiral symmetry breaking phase transition at strong coupling, and was not a candidate for a relativistic continuum quantum field theory [1]. The noncompact formulation of the model was seen to have a second order chiral transition which became a candidate for a continuum field theory [2]. However, since the transition occurs at non-zero coupling conventional classical or perturbatively-improved Actions do not necessarily yield reliable pictures of the theory's critical point. In fact, monopoles were discovered near the chiral transition and large scale simulations suggested that a topological phase transition, monopole condensation, occurs at the chiral transition [3]. This interesting result, which will be strengthened considerably through additional simulations and finite size scaling analyses here, means that conventional perturbative discussions of the triviality of QED are not relevant here [4]. Theories with fundamental monopoles are intrinsically nonperturbative because of the Dirac quantization condition. Studies of the dynamical nature of theories with fundamental electric and

magnetic charges [5] are completely new even though the subject is fifty years old [6]. Recent simulations have suggested that the essential characteristics of the critical point Action driving the physics we are observing in simulations includes at least three ingredients: 1) a strong vector force; 2) induced four Fermi couplings; and 3) monopole condensation. Item 2 has been discussed and motivated elsewhere [7,8] and will not be discussed explicitly here. Certainly, considering the richness of items 1-3, this particular study is, alas, still in its infancy. Nonetheless, the article will present promising mass spectrum calculations which, when combined with accurate chiral condensate measurements, will set the stage for decisive critical exponent and anomalous dimension calculations which should help clarify the theory's critical Action.

In Sec. 2 we introduce a random refreshment procedure to make the hybrid molecular dynamics algorithm more efficient. In Sec.3 we shall present data for the chiral condensate which suggests the existence of a non-trivial phase transition at a coupling $\beta_{c}=0.225(5)$. Since we have previously done a similar study on a $10^{4}$ lattice, we shall monitor possible finite size effects carefully. In Sec.4 we study monopole condensation in this theory and find that there is a phase transition at $\beta_{c}=0.225$ whose singularities are compatible with four dimensional percolation. In Sec.5 we consider the meson mass spectrum of the model. A study of the 
ratio of the pion and sigma masses is consistent with a power-law chiral phase transition at $\beta_{c}=0.225(5)$ with critical indices agreeing with those found in the Equation of State study of Sec.3. An analysis of the dependence of the pion mass on the chiral condensate also picks out $\beta_{c}=0.225$ and suggests that the transition is non-trivial. And finally, the systematics of other meson mass ratios are studied and the level ordering of the theory is presented. In Sec.6 of this paper we present suggestions for further study, both computational and analytic.

\section{A Random Refreshment Algorithm}

The lattice Action and simulation algorithm have been presented and discussed in great detail in past work. The reader is referred to Ref.[9] for explicit formulas. As will be discussed below, the hybrid molecular dynamics algorithm was considerably improved by introducing "random refreshing". This improvement tended to decorrelate low momentum photon modes in our gauge configurations more efficiently than in past studies. To control systematic errors we were forced to use a small time step in the molecular dynamics evolution equations. In fact, on $16^{4}$ lattices we accumulated data with bare fermion masses $\mathrm{m}$ of 0.03 , 0.02 , 0.01 and 0.005 in lattice units. The time step ranged from 0.02 at $m=0.03$ to 0.005 at $m=0.005$ to keep systematic errors smaller than statistical ones. The criterion for the convergence of the conjugate gradient algorithm used to invert the lattice Dirac operator was scaled with lattice size and fermion mass to maintain uniform accuracy throughout our data set. At each coupling $\beta=1 / e^{2}$ and $m$ value we generated between 200 and 400 molecular dynamics trajectories. Each trajectory was defined to be one time unit long, so 400 trajectories at $m=0.005$, where the time step was 0.005 , corresponded to 80,000 sweeps of the hybrid algorithm. We collected data at couplings ranging from $\beta=0.15$ (strong coupling) to $\beta=0.30$ (weak coupling). This study dwarfs all others in the literature. Nonetheless, our meson spectrum data is not as accurate as we would have liked. In the last section of this paper we shall suggest further improvements on the present work to generate more plentiful useful results.

In performing our updating in the weakly coupled spin wave phase we observed that the time development of the gauge field was very similar to that for the free gauge field, although fermion feedback shifted the chiral symmetry breaking transition to a considerably smaller value of the coupling $\beta=1 / e^{2}$. The normal refreshment scheme is inadequate for such dynamics. We have therefore developed a random refreshment scheme which is adapted for the free field case and hence should be better than the standard scheme for the problem at hand. The free field lagrangian from which we start is

$$
L=12 \sum_{\text {links }} \dot{\Theta}_{\mu}^{2}-4 \beta \sum_{\text {sites }, \mu, \nu}\left(\Delta_{\mu} A_{\nu}-\Delta_{\nu} A_{\mu}\right)
$$

In momentum space, using the Feynman gauge for convenience, $L$ becomes

$$
L=\sum_{k=2 \pi n / L}\left(12 \dot{\Theta}_{\mu}^{2}(k)-\beta\left(4-\sum_{\nu} \cos k_{\nu}\right) A_{\mu}^{2}(k)\right)
$$

i.e. a set of harmonic oscillators with frequencies 


$$
\omega_{k}=\sqrt{2 \beta\left(4-\sum_{\nu} \cos k_{\nu}\right)}
$$

so the periods $\tau_{k}$ of these oscillators lie in the range

$$
\frac{\pi}{2 \sqrt{\beta}} \leq \tau_{k} \leq \frac{2 \pi}{\sqrt{2 \beta(1-\cos (2 \pi / L))}}
$$

For $\beta=0.225$ where the transition occurs on a $16^{4}$ lattice this range is $3.3-33.9$.

Ideally for any one of these oscillators, one would like to refresh often enough so that it samples its own (gaussian) energy distribution as rapidly as possible and loses any phase coherence with the other oscillators. However, we do not want to refresh it so often that it only random walks through its own configuration space. Our educated guess is that the best choice would be a refreshment interval $\sim 1 / \omega_{k}[10]$. If we were to choose this for the fastest oscillator, the slowest oscillator would random walk through its configuration space, while if we chose this for the slowest oscillator the fastest oscillator would spend most of its time retracing its orbit. We thus chose a compromise where the refreshment interval is varied randomly to cover the "optimal" intervals for each of the oscillators. In fact we choose the refreshment interval each time to vary between $\tau_{\min }$ and $\tau_{\max }$ according to

$$
\tau_{\text {refresh }}=\tau_{\min }\left(\tau_{\max } / \tau_{\min }\right)^{R}
$$

where $R$ is chosen from a set of random numbers distributed uniformly over the interval [0,1]. For our runs we chose $\tau_{\min }=0.2$ and $\tau_{\max }=10$. For runs somewhat longer than ours $(\sim 250$ time units per $\beta)$ we would suggest increasing $\tau_{\max }$ closer to the maximum period of the oscillators.

\section{Chiral Equation of State}

The data for the average plaquette and $\left\langle\bar{\psi} \psi>\right.$ at various couplings $\beta=1 / e^{2}$ and bare fermion masses $m$ are presented in Tables 1 and 2, respectively. The $\langle\bar{\psi} \psi>$ data is plotted in Fig. 1. A particularly useful, relatively unbiased way to extract physics from these measurements it to construct the theory's equation of state [11],

$$
\frac{<\bar{\psi} \psi>}{m^{1 / \delta}}=F\left(\frac{\Delta \beta}{<\bar{\psi} \psi>^{1 / \beta_{m a g}}}\right)
$$

where $\Delta \beta=\beta-\beta_{c}, \beta_{c}$ is the bulk critical point for chiral symmetry restoration, and $\delta$ and $\beta_{\text {mag }}$ are critical exponents. At $\beta=\beta_{c}, \delta$ records the response of the order parameter to a small symmetry breaking field,

$$
<\bar{\psi} \psi>=C m^{1 / \delta}
$$

while $\beta_{\text {mag }}$ controls the shape of the $\langle\bar{\psi} \psi>$ vs. $\beta$ curve in the broken phase in the chiral limit $m \rightarrow 0$,

$$
<\bar{\psi} \psi>=D\left(\beta_{c}-\beta\right)^{\beta_{m a g}}
$$


Eq.(3.3) is difficult to use in practice because it refers to $\langle\bar{\psi} \psi>$ at vanishing fermion mass $m$, a quantity which is not directly accessible to our algorithm. Our strategy will then be to exploit Eq. (3.2) to find candidates for $\beta_{c}$ and $\delta$, and then construct the equation of state Eq.(3.1) for various $\beta_{\text {mag }}$. Only for the correct, physical values of $\beta_{\text {mag }}$ will all the data of Table 2 fall on the universal curve Eq. (3.1). Past studies on smaller lattices and polynomial extrapolations of $\langle\bar{\psi} \psi>$ to zero $m$ at fixed coupling $\beta$, indicate that the critical coupling is near 0.225 and $\beta_{\text {mag }}$ is between 0.50 and 1.00 [3]. To search for the power-law behavior of Eq. (3.2) expected at the critical point, we plot $-\ln \langle\bar{\psi} \psi>$ vs. $-\ln m$ for $m=0.005,0.01$ and 0.02 in Fig. 2. Note that at $\beta=0.22$, the $\langle\bar{\psi} \psi>$ value at $m=0.005$ is larger than the power-law fit to the $m=0.01$ and 0.02 data points, while at $\beta=0.225$ it is smaller than the fit. This suggests that $\beta=0.220$ lies in the broken symmetry phase and the critical coupling is between 0.220 and 0.225 . The straight line fits in Fig. 2, then predicts that the critical index $\delta$ lies between 2.31 and 2.75. The reader might recall that this procedure was extremely successful for the quenched model where much greater statistics and smaller quark masses could be probed [12]. In that case data was taken with a resolution of 0.001 in $\beta$ rather than 0.005 used here and the critical coupling was determined to three significant digits.

It is also interesting to search for $\beta_{c}$ and $\delta$ in a slightly different way. Consider the plots of $-1 / \ln (m)$ vs. $-1 / \ln \langle\bar{\psi} \psi>$ at fixed $\beta$ in Fig.3. This fit is particularly suitable for determining the position of the critical coupling and the value of the critical exponent $\delta$. Unlike the previous plot, it brings the chiral limit, $m=0$, to the origin. Consider the EOS in its standard form [11], $m=<\bar{\psi} \psi>^{\delta} f(z)$, with $z$ being the reduced variable as defined by Eq.(3.1). The universal function $f(z)$ is monotonically decreasing with only one zero in the broken phase. Denote $x=-1 / \ln \langle\bar{\psi} \psi>$ and $y=-1 / \ln (m)$. Then,

$$
y=\frac{x}{\delta-x \ln f(z)}
$$

Because $f(z)$ vanishes in the chiral limit in the broken phase, the curve $y=y(x)$ approaches the horizontal axis with an infinite slope, while in the symmetric phase it approaches the origin linearly, with unit slope. At the critical point $z=0$ we have,

$$
y_{c}=\frac{x}{\delta-x \ln f(0)}
$$

As we will see in later sections, the universal function $f(z)$ will turn out to satisfy $f(0) \approx 1$ so that the second term in the numerator of Eq.(3.4b) can be neglected and the slope of the curve $y_{c} \approx x / \delta$ gives a fairly good estimate of the exponent $\delta$. From these two equations it follows that

$$
\frac{1}{y_{c}}-\frac{1}{y}=\ln \frac{f(z)}{f(0)}
$$

Considering the monotonicity of the function $f(z)$, the difference $y(x)-y_{c}(x)$ changes sign as the critical point is crossed. All the curves in the broken phase lie below, and those in the symmetric phase lie above the critical line. The lines lying in the broken phase terminate on the horizontal axis, whereas those in the symmetric phase and at the critical point terminate at the origin. Except at the critical point, all the lines have curvature downwards. From the figure we see that the straight line hypothesis works well at $\beta=0.225$ where it gives $\delta=2.29$ in good agreement with Fig. 2. In addition, the curves for $\beta=0.22$ and 0.210 suggest 
that $\langle\bar{\psi} \psi>$ is non-zero in the chiral limit at $m \rightarrow 0$, while the curves for $\beta=0.23,0.24$ and 0.26 indicate that $<\bar{\psi} \psi>$ vanishes in the chiral limit.

Consider the equation of state Eq.(3.1) for different hypothetical $\beta_{c}$ values $0.230,0.225$ and 0.220 . For each $\beta_{c}$ and $\delta$ value we can inspect Eq.(3.1) as a function of $\beta_{\text {mag }}$. We will show just three characteristic plots from the multitude we viewed. Recall the definition of the susceptibility critical index $\gamma, \beta_{\text {mag }}=\gamma /(\delta-1)$. We shall show numerical evidence for the equality $\gamma=1$ later in this article. Recall also that $\gamma$ is exactly unity in the quenched model as well as in mean field theories of chiral transitions. So, we will investigate the hypothesis $\gamma=1$ and show in Figs. 4, 5 and 6, the chiral equation of state for the three cases: (1) $\beta_{c}=0.220, \delta=2.75, \beta_{\text {mag }}=0.571,(2) \beta_{c}=0.225, \delta=2.31, \beta_{\text {mag }}=0.763$, and $(3) \beta_{c}=0.230, \delta=1.89$, $\beta_{\text {mag }}=1.124$. The universal character of the plots - that all the data at various couplings and quark masses fall on one curve - is fairly good. Inspecting the three figures, we see that the dispersion in the $\beta_{c}=0.225$ case is the least, and this fact suggests that

$$
\beta_{c}=0.225, \quad \delta=2.31, \quad \beta_{\text {mag }}=0.763
$$

as our "preferred" conclusion from these measurements.

These $16^{4}$ results should be contrasted with our $10^{4}$ results published earlier [3]. Comparing the raw $<\bar{\psi} \psi>$ data between the two lattice sizes, we note considerably more precision in our present study which accounts for the fact that we have stronger conclusions here. Our preferred values for $\beta_{c}, \delta$ and $\beta_{\text {mag }}$ as listed in Eq.(3.6) are compatible with our $10^{4}$ results. The only significant finite size effects in our chiral condensate measurements occur at the smallest $m=0.005$ value where the $\langle\bar{\psi} \psi>$ values on the larger lattice lie above those on the smaller lattice. This is the expected systematic trend - the smaller lattice acts as a "finite temperature" environment, suppressing chiral symmetry breaking. Note that at $\beta_{c}=0.225$, $<\bar{\psi} \psi>=0.0881(29)$ on a $10^{4}$ lattice and $<\bar{\psi} \psi>=0.0943(8)$ on a $16^{4}$ lattice which is a $6 \%$ shift ignoring error bars and a two standard deviation effect otherwise.

We shall see in Sec.5.b below that meson spectroscopy also favors $\beta_{c}=0.225$ and $\delta=2.31$ in a completely independent fashion.

\section{Monopole Condensation}

In past work we have identified $\beta_{c}=0.225$ as the critical point for monopole condensation, using order parameter and susceptibilities first suggested in Ref.[13]. We can now sharpen that analysis using finite size scaling analyses to argue that monopole condensation is a true phase transition with power-law thermodynamic singularities. The precise definition of bond percolation and the identification of monopoles within a gauge field configuration can be found in Ref.[13] and will not be repeated here. Our order parameter for the monopole condensation transition will be $M=n_{\max } / n_{\text {tot }}$ which is the ratio of the number of dual sites in the largest monopole cluster to the total number of connected sites in all monopole clusters. Fluctuations in $M$ generate a monopole susceptibility $\chi$ which should diverge at the critical point. In Fig.7 we show $\chi$ vs. $\beta$ on the $16^{4}$ lattice for bare quark masses of $0.03,0.02$ and 0.01 . There is no systematic dependence on the quark mass over this range of relatively small values. The critical point for monopole condensation is seen to be $\beta_{c}=0.225(5)$. Recall that in the quenched noncompact lattice QED model $\chi$ peaked at weaker 
coupling $\beta=0.244$, so charge screening has pushed the monopole condensation point to stronger coupling as expected [14]. However, the peak values of $\chi$ are within error bars in the two cases. Finite size scaling relates the maximum value of $\chi, \chi_{\max }(L)$, on a lattice of volume $L^{4}$ to the critical indices $\gamma_{\text {mon }}$ and $\nu_{\text {mon }}$ of the monopole condensation transition,

$$
\chi_{\max } \sim L^{\gamma_{\operatorname{mon}} / \nu_{\operatorname{mon}}}
$$

In Fig. 8 we show $\ln \chi_{\max }$ plotted against $\ln L$ for $\beta_{c}=0.225$ for the data presented in Table 3. For each lattice size we confirmed that $\beta_{c}=0.225(5)$ - no size dependence was observed in the critical point of this transition (This peculiar fact was also observed in the quenched case [14].). Note that a linear fit to Fig.8 is very good and we read off the ratio of the critical indices $\gamma_{m o n}$ and $\nu_{m o n}$,

$$
\gamma_{m o n} / \nu_{m o n}=2.16(6)
$$

In the quenched case we measured $\gamma_{\text {mon }} / \nu_{\text {mon }}=2.24(2)$.

Now consider the behavior of the order parameter $M=n_{\max } / n_{\text {tot }}$. First consider histograms of $M$ on the $16^{4}$ lattice at $m=0.01$ and variable $\beta$. In Fig.9a we consider weak coupling $\beta=0.24$ and find that $M$ is essentially zero with little dispersion. However, at $\beta=0.23$ shown in Fig.9b, the distribution has moved to positive $M$ and has broadened. At $\beta=0.225$ shown in Fig.9c the effect is most dramatic. Continuing to $\beta=0.22$ (Fig.9d), $\beta=0.21$ (Fig.9e) and $\beta=0.20$ (Fig.9f), we see the order parameter increase in value and its fluctuations diminish. A plot of $M$ vs. $\beta$ will also be presented below.

A particularly effective way to measure the "magnetic" critical exponent $\beta_{m o n}$,

$$
M(\beta)=C\left(\beta_{c}-\beta\right)^{\beta_{m o n}}, \quad\left(\beta<\beta_{c}\right)
$$

which controls the nonanalytic vanishing of $M$ in the $L$ limit, is to use finite size scaling. At the critical point and on a $L^{4}$ lattice $M\left(\beta_{c}, L\right)$ should vanish with a power-law,

$$
M\left(\beta_{c}, L\right) \sim L^{-\beta_{m o n} / \nu_{m o n}},
$$

Using the data in Table 3 we show $\ln M\left(\beta_{c}, L\right)$ vs. $\ln L$ in Fig.10 for $L$ ranging from 10 to 18. The error bars are much larger than we would prefer, but an estimate of $\beta_{m o n} / \nu_{m o n}$, follows from the $L=12,14,16$ and 18 points:

$$
\beta_{\text {mon }} / \nu_{\text {mon }}=0.64(22)
$$

Similar measurements in the quenched model [14] gave $\beta_{\text {mon }} / \nu_{\text {mon }}=0.88(2)$. The values of $M\left(\beta_{c}, L\right)$ for the quenched model were systematically below those of Table 3. It is not clear whether fermion feedback has reduced $\beta_{\text {mon }} / \nu_{\text {mon }}$, below its quenched value since the error bars in Fig.10 are relatively large.

In Ref.[14] we argued that monopole condensation in quenched noncompact lattice QED is a percolation process uncomplicated by dynamical considerations. Our perspective on this problem comes from bond percolation models where one randomly occupies bonds with a probability $\rho$. If $\rho$ is small, typical lattice configurations have small, improbable connected clusters of occupied bonds. However, as $\rho$ increases towards 
a critical concentration $\rho_{c}$, the largest connected cluster becomes infinite in extent and occupies a macroscopic fraction of the available lattice bonds. The value $\rho_{c}$ is called the percolation threshold and traditional statistical mechanics calculations produce the estimate $\rho_{c} \approx 0.16$ in four dimensions. Following Ref.[13] we computed the total amount of monopole current $\ell, \ell=\sum_{n, \mu}\left|m_{\mu}(\tilde{n})\right|$, where $m_{\mu}(\tilde{n})$ is the integer-valued monopole current defined on links of the dual lattice, in each of our $16^{4}$ configurations. The resulting curve of the average value of $\ell$ normalized by the number of lattice links $N_{\ell}$ is shown in Fig.11. We also show the percolation order parameter $M=n_{\max } / n_{\text {tot }}$ there for the same set of configurations having small $m=0.01$. We see that $M$ turns on at $\beta=0.225$ with the expected finite size rounding and that $\left\langle\ell>/ N_{\ell}\right.$ is roughly 0.135 and varies smoothly around the critical point. We learn that $\left\langle\ell>/ N_{\ell}\right.$ is near the critical concentration of pure bond percolation and since it varies smoothly it could be used as an independent variable to parameterize the monopole measurements instead of the gauge couplings $\beta$. It is interesting that $\langle\ell\rangle / N_{\ell}$ varies abruptly across the confinement transition of pure compact U(1) lattice gauge theory [15]. The character of Fig.11 suggests that the monopole dynamics of the two flavor noncompact model is not qualitatively different from the quenched model where accurate finite size scaling measurements placed the monopole transition in the universality class of four dimensional percolation [14].

In summary, the two flavor theory has a monopole condensation transition at $\beta_{c}=0.225$ with powerlaw thermodynamic singularities. The determination of $\gamma_{m o n} / \nu_{m o n}$ is in good agreement with the same calculation for the quenched model which in turn has critical indices in agreement with four dimensional percolation [16]. The measurement of $\beta_{\text {mon }} / \nu_{m o n}$ is less quantitative, but the data for $M=n_{\max } / n_{\text {tot }}$ certainly satisfies a scaling hypothesis. It is tempting to conclude that the chiral symmetry breaking transition and the monopole condensation transition are coincident. If that is true, then the set of field theory critical indices controlling the chiral transition $\left(\delta=2.31, \beta_{\text {mag }}=0.763\right)$ would be distinctly different from mean field values.

It is sometimes said that the monopoles of lattice QED are artifacts of the regularization scheme and have no physical significance. However, in this case we have seen that they experience a second order phase transition with scaling laws and critical indices which satisfy hyperscaling. Therefore, their critical properties have decoupled from the lattice itself and they should have macroscopic physical consequences.

\section{Spectrum}

\section{a. Raw Data}

We calculated the propagators of the pion, sigma, rho, and $A_{1}$ mesons using standard techniques borrowed from lattice QCD large scale simulations [17]. Most of the meson propagator calculations used a point source for the quark propagator calculation and then took the zero momentum projection of the propagator perpendicular to the "temporal" direction of the $16^{4}$ lattice. The fall-off of the meson propagator in the temporal direction then determined the mass of the state using fitting technology borrowed from lattice QCD [17]. The standard parameterization reads,

$$
G(t)=\sum_{i} C_{i}\left(\exp \left(-M_{i} \tau\right)+\exp \left(-M_{i}(16-\tau)\right)\right)+\sum_{i}(-1)^{\tau} D_{i}\left(\exp \left(-\tilde{M}_{i} \tau\right)+\exp \left(-\tilde{M}_{i}(16-\tau)\right)\right)
$$


and we considered fits over different ranges of $\tau$ between 0 and 8. In most cases good fits were found with just the leading single particle term in Eq.(5.1) determining the amplitude $C$, and the mass $M$, but in several cases secondary particles were also necessary although their masses and amplitudes were not determined with sufficient accuracy to be interesting. Error estimates on the masses followed from the usual correlated $\chi^{2}$ procedures, also borrowed from lattice QCD studies [17].

Before turning to the masses themselves, it is instructive to consider typical fits. Consider the coupling $\beta=0.225$ and $m=0.01$, and the pion (Fig.12), sigma (Fig.13), rho (Fig.14) and $A_{1}$ (Fig.15) propagators. We show both the raw data (open circles) and the fits (triangles). As usual, the fits for the pion were the best, and we will be able to do some quantitative analysis with the data here. The sigma particle also admitted respectable fits and its mass estimates will be useful. The rho and $A_{1}$ were relatively disappointing and very little quantitative information will be obtained in these cases.

The data of meson masses (squared) for couplings ranging from 0.20 to 0.26 and quark masses from 0.03 to 0.005 are given in Tables 4, 5, 6 and 7 . The quality of the fits is indicated in part by the error bars accompanying each entry. Typically, especially in the broken symmetry phase, the confidence levels of the fits were very good ( $90 \%$ and higher confidence levels were common). However, the quality of the fits in the weak coupling phase was distinctly poorer. The simple hypothesis Eq.(5.1) is probably too naive in the weak coupling phase where light charged fermions propagate freely, interacting with massless photons, etc. This result is certainly not very surprising, but we did not know better fitting forms than Eq.(5.1) which could be used productively on our limited data set. In addition, examining the rho and $A_{1}$ tables, we see that the large statistical errors in the data renders the fits of little use. Missing entries in the tables indicate that the fitting routine was unable to find an acceptable fit for that particular propagator. Clearly it is meaningless to extrapolate the rho and $A_{1}$ masses to $m \rightarrow 0$. Therefore, our analysis will consider $m=0.01$ for the most part for these two states. Happily, the pion and sigma results are much more quantitative and we will learn a lot from their $m$ and $\beta$ dependencies.

\section{b. The Goldstone Pion}

If chiral symmetry is really broken at $\beta_{c}=0.225$, then the pion should be massless in the strong coupling phase. This effect is quite clear in the data. If we extrapolate the pion mass-squared to $m=0$, zero symmetry breaking, then Fig.16 follows. The $m \rightarrow 0$ extrapolation was done using the $m=0.03,0.02$ and 0.01 data and the form

$$
M_{\pi}^{2}=a+b m+c m^{2}+\ldots
$$

expected from PCAC. Although the error bars in Fig.16 are quite large, $M_{\pi}^{2}$ is compatible with zero for $\beta \leq 0.225$ and is non-zero in the weak coupling phase. Recall, however, our caveat about the fitting procedure Eq.(5.1) at weak coupling the simple pole structure of composite meson propagators in momentum space is probably too naive in the weak coupling phase to parameterize the physics quantitatively. Nonetheless, the qualitative fact that the pion mass is distinctly non-zero in the weakly coupled phase is confirmed by the simulation. Chiral symmetry restoration in the weak coupling phase will be discussed in greater detail below. 
c. Spectroscopy and the Critical Index $\delta$

We have argued in Ref.[18] that the properties of ratios of dynamically generated masses $M_{a}$ and $M_{b}$, $\left.R_{a b}(m, \beta)=G\left(m /\left(\beta-\beta_{c}\right)^{\Delta}\right)\right)$, with $\Delta=\delta \beta_{\text {mag }}$, are determined by the Equation of State of the chiral order parameter. Let $R(m, \beta)$ denote the ratio of $M_{\pi}^{2}$ to $M_{\sigma}^{2}$ and write the Equation of State in the standard form,

$$
m=<\bar{\psi} \psi>^{\delta} f\left(\frac{\Delta \beta}{<\bar{\psi} \psi>^{1 / \beta_{m a g}}}\right)
$$

with $\Delta \beta=\beta_{c}-\beta$. Then [18],

$$
\frac{1}{R(m, \beta)}=\delta-\frac{x}{\beta_{m a g}} \frac{f^{\prime}(x)}{f(x)}
$$

where $x$ is the reduced coupling, $\Delta \beta /<\bar{\psi} \psi>^{1 / \beta_{m a g}}$. Eq.(5.4a) reduces at the critical point $\beta=\beta_{c}(x=0)$ to,

$$
R\left(m, \beta_{c}\right)=\frac{M_{\pi}^{2}}{M_{\sigma}^{2}}=\frac{1}{\delta}
$$

This result will give us another measurement of $\beta_{c}$ and $\delta$ to supplement and, in fact, support Eq. (3.6). Recall that the derivation of Eq. (5.4a-b) follows from the chiral equation of state describing the spontaneous symmetry breaking of a continuous group [18]. In fact, the derivation reveals more than Eq. (5.4b) which states that at the critical point the ratio of the pion and sigma masses squared is independent of $m$ and is equal to $1 / \delta$. For fixed $\beta<\beta_{c}$ in the broken symmetry phase, $R(m, \beta)$ should fall to zero as $m \rightarrow 0$ since the pion is a Goldstone boson. For fixed $\beta>\beta_{c}$ in the symmetric phase, $R(m, \beta)$ should increase to unity as $m \rightarrow 0$ since the sigma and pion must be degenerate in the symmetric phase. Thus, in the scaling region, the following bound can be placed on $\delta[18]^{\dagger}$

$$
R\left(m, \beta \leq \beta_{c}\right) \leq \frac{1}{\delta} \leq R\left(m, \beta \geq \beta_{c}\right) .
$$

Because of the scaling form of the mass ratio, this bound improves as either $\beta$ approaches $\beta_{c}$ or as $m$ becomes large.

In Fig.17 we plot $R(m, \beta)$ using the data for $M_{\pi}^{2}$ and $M_{\sigma}^{2}$ in Tables 4 and 5. For $\beta=0.20,0.21$ and $0.22 R$ clearly falls as $m$ decreases while for $\beta=0.23,0.24$ and $0.25 R$ increases as $m$ decreases. Only at $\beta=0.225$ is $R$ independent of $m$ and has the value $0.391(11)$ which gives

$$
\delta=2.56(7)
$$

The consistency of these results with the analysis based on the chiral equation of state in Sec.3 is quite striking. In fact, the determination of $\beta_{c}=0.225(5)$ is most persuasive from Fig. $17-R(m, \beta)$ systematically decreases with $m$ at $\beta=0.22$ and systematically increases as $m$ decreases at $\beta=0.23$, thus picking out $\beta_{c}=0.225(5)$ as the critical point.

$\dagger$ An analysis very similar to ours was used to determine the universality class of the finite temperature chiral restoration transition in QCD at infinite couplings by, G. Boyd, J Fingberg, F. Karsch, L Kärkkäinen and B. Peterson, Nucl. Phys. B376, 199 (1992). 
It is interesting to pursue Eq. (5.3) and (5.4) in somewhat greater detail. In particular, Eq. (5.4a) states that the mass ratio $R=M_{\pi}^{2} / M_{\sigma}^{2}$ follows from the Equation of State with no additional parameters, etc. Do our chiral condensate measurements produce a universal Equation of State $f(x)$ which predicts the correct systematics for $R$ ? This is a highly nontrivial test of the hypothesis that there is a chiral critical point at $\beta_{c}=0.225$ with $\delta=2.31$ and $\beta_{m a g}=0.763$ since the spectroscopy measurements of $M_{\pi}^{2}$ and $M_{\sigma}^{2}$ were independent of chiral condensate considerations. In Fig.18 we plot the Equation of State in the "standard" form Eq. (5.3). One of the advantages of plotting the Equation of State in this form is that the universal function $f$ is so simple. Clearly a linear fit for $f$ represents the data adequately. In fact, as discussed in Ref.[18] a linear $f$ occurs only in a theory where the susceptibility index $\gamma$ is unity. As mentioned in our discussion of Fig. 4-6 and now seen in Fig.18, the simulation data does provide direct evidence for $\gamma=1$. The interested reader is directed to Ref.[18] for further detail, since here our emphasis is on spectroscopy and the exponent $\delta$. A linear fit to $f(x)$ gives,

$$
f(x)=-5.3125 x+1.075
$$

Using Eq.(5.4a) $R(m, \beta)$ is now determined in a universal form and is plotted in Fig.19. Comparing Fig.17 and Fig.19 we find good agreement within the rather substantial error bars for our spectroscopy measurements. Nonetheless, the general success of Eq.(5.3) and (5.4) to describe spectroscopy data within the hypothesis of a chiral critical point with power-law non-mean field singularities is encouraging.

d. $M_{\pi}^{2}$ vs. $<\bar{\psi} \psi>^{2}$, the Critical Point and Anomalous Dimensions

As we have discussed in Ref.[18], measurements of the pion mass and the chiral order parameter can be exploited in unison to locate critical points and measure anomalous dimensions. The two theoretical ingredients underlying this strategy is 1) correlation scaling; and 2) the equation of state. In addition, the Goldstone nature of the pion, which we have confirmed explicitly above, allows additional information to be extracted from plots of $M_{\pi}^{2}$ vs. $\left\langle\bar{\psi} \psi>^{2}\right.$. We refer the interested reader to Ref.[18] for derivations. Here it is more appropriate to quote results and apply them to our simulation data. It is convenient to express the results in terms of $\eta$, the anomalous dimension of $\langle\bar{\psi} \psi\rangle$. According to hyperscaling the anomalous dimension $\eta$ is related to the critical indices $\beta_{\text {mag }}$ of the order parameter and $\nu$ of the correlation length by $\beta_{\text {mag }} / \nu=1+\eta / 2$. Ref.[18] predicts the squared pion mass' dependence on the square of the chiral order parameter within the scaling region for fixed coupling $\beta$. Consider the broken symmetry phase $\beta<\beta_{c}$, the critical point $\beta=\beta_{c}$ and the symmetric phase $\beta<\beta_{c}$ in turn,

$$
\begin{array}{ll}
\beta<\beta_{c}: & M_{\pi}^{2} \sim<\bar{\psi} \psi>^{2}-<\bar{\psi} \psi>_{0} \\
\beta=\beta_{c}: & M_{\pi}^{2} \sim\left(<\bar{\psi} \psi>^{2}\right)^{1 /(1+\eta / 2)} \\
\beta>\beta_{c}: & M_{\pi}^{2} \sim\left(<\bar{\psi} \psi>^{2}\right)^{1 /(1+2 / \eta)}
\end{array}
$$

So, Eq.(5.8a) states that $M_{\pi}^{2}$ should vanish linearly as $m \rightarrow 0$ and $\left\langle\bar{\psi} \psi>^{2} \rightarrow<\bar{\psi} \psi>_{0}^{2} \neq 0\right.$. Eq.(5.8b) states that at the critical point $M_{\pi}^{2}$ vs $<\bar{\psi} \psi>^{2}$ should be concave downward and provide a measurement of the anomalous dimension $\eta$. Recall that a physically meaningful theory must have $\eta>0$, so $1 /(1+\eta / 2)<1$. Finally, Eq.(5.8c) states that the curves in the symmetric phase will also be concave downward and will be 
sensitive to $\eta$. Clearly, Eq.(5.8c) does not apply in the case $\eta=0$. The more general formulas of Ref.[18] show that in a mean field scenario where $\eta=0$ that $M_{\pi}^{2}$ should vary as $C\left(\beta-\beta_{c}\right)+<\bar{\psi} \psi>^{2}$ in the symmetric phase.

In summary, we learn from Eq.(5.8) that plots of $M_{\pi}^{2}$ vs. $<\bar{\psi} \psi>^{2}$ are sensitive probes into the dynamics of the critical point. For example, if mean field theory applies then the $M_{\pi}^{2}$ curves should be linear functions of $\left\langle\bar{\psi} \psi>^{2}\right.$ for all coupling and the curve which passes through the origin picks out the critical point uniquely. If an interacting field theory describes the transition, then the $M_{\pi}^{2}$ curves should be linear only in the broken symmetry phase and otherwise have downward curvature determined by the anomalous dimension $\eta$.

In Fig.20 we present our $M_{\pi}^{2}$ and $\left\langle\bar{\psi} \psi>^{2}\right.$ data in this format. Clearly the curves are linear for $\beta \leq 0.220$ and give a non-zero chiral condensate in the chiral limit $M_{\pi}^{2} \rightarrow 0$. The functional dependence of the $\beta=0.225$ and 0.230 curves is not decisively predicted, while the $\beta=0.240$ and 0.250 curves are concave downward. We obtain an estimate of $\beta_{c}=0.225-0.230$ in good agreement with our other approaches and, although our data is not good enough to yield a useful value for $\eta$, we obtain qualitative evidence that the critical point is not described by a mean field theory.

e. Mass Ratios and Chiral Symmetry Restoration at Weak Coupling

One of our aims in these simulations is the determination of meson mass ratios in the chiral limit. As shown in our discussion of $M_{\pi}^{2} / M_{\sigma}^{2}$ these quantities can indicate whether the chiral critical point in trivial and teach us about the realization of chiral symmetry in the two phases. Unfortunately our rho and $A_{1}$ data are not quantitative enough to use in a similar fashion. Therefore, we shall only consider $m=0.01$ data here and look for trends which we believe should survive the $m \rightarrow 0$ extrapolation.

First consider the ratios $M_{\pi}^{2} / M_{\sigma}^{2}$ and $M_{\pi}^{2} / M_{\rho}^{2}$ at variable coupling $\beta$. In Fig.21 we present the results which indicate that both ratios have non-zero values in the weak coupling phase. In fact $M_{\pi}^{2} / M_{\sigma}^{2}$ rapidly increases to $0.915(15)$ at $\beta=0.26$. We expect $M_{\pi}^{2} / M_{\sigma}^{2}=1$ in a phase where chiral symmetry is not spontaneously broken, so this is a welcome result. Looking back at Fig.17 we see $R(m, \beta)$ rapidly rising toward unity as $m \rightarrow 0$ for $\beta$ values of 0.24 and 0.25 , so this particular result looks rather firm. Note from Fig.21 that $M_{\pi}^{2} / M_{\rho}^{2}$ appears to be non-zero in the weak coupling phase but its particular value is not related to symmetry considerations.

Next, consider the ratio $M_{\rho}^{2} / M_{A_{1}}^{2}$ at $m=0.01$ for various couplings as plotted in Fig.22. At weak coupling, $\beta=0.26$ say, the ratio is consistent with unity, which is the expected value in a phase where chiral symmetry is an unbroken symmetry,

$$
\frac{M_{A_{1}}^{2}}{M_{\rho}^{2}}=0.96(8) \quad \text { (weak coupling) }
$$

As we pass to the strong coupling phase this ratio has certainly decreased, but its precise value is obscured by large error bars. The data suggests considerable splitting in the strong coupling phase,

$$
\frac{M_{A_{1}}^{2}}{M_{\rho}^{2}} \approx 1.35 \quad \text { (broken phase) }
$$

but we hesitate to place an error bar on Eq.(5.10). 
The ratio $M_{\sigma}^{2} / M_{\rho}^{2}$ at $m=0.01$ varies much less dramatically as $\beta$ is varied. In the vicinity of the critical point and at weak coupling, Fig.23 shows that

$$
\frac{M_{\sigma}^{2}}{M_{\rho}^{2}} \approx 0.35
$$

A similar impression comes from Fig.24 where $M_{\sigma}^{2} / M_{\rho}^{2}$ is plotted against $M_{\pi}^{2} / M_{\rho}^{2}$ but the figure is obscured by large error bars.

\section{Conclusions and Prospects}

Instead of summarizing our results, we shall end with a few words about future work. It should be clear to the reader, however, that considerable progress has been made in understanding the two flavor version of noncompact lattice QED and its chiral transition. That transition appears to be characterized by non-mean field exponents and monopoles probably play an important role in its existence. The use of the Equation of State and spectroscopy data appears to be a particularly productive approach towards establishing its physical characteristics.

Obviously we must confirm these results on larger lattices and push our finite size scaling studies further before solid claims can be made about the continuum limit of the lattice theory. Spectroscopy studies using wall sources should be made on asymmetric lattices with ten times the statistics accumulated here. A serious simulation on $64 \times 32^{3}$ lattices seems essential.

The physical basis of the chiral transition at $\beta_{c}=0.225(5)$ must be clarified. We need to obtain a quantitative understanding of the importance of monopoles at the transition. We have already begun a project in that direction. By cooling lattice configurations already generated here, we can produce configurations with the monopoles intact, but the photons removed. Is $\langle\bar{\psi} \psi\rangle$ the same in these cooled configurations for $\beta<0.225$ ? Additional calculations of this variety may clarify the content of the lattice theory's critical Action. Interesting work in this area has already appeared [19]. What is the fate of the fermion and the photon in the broken symmetry phase? Our attempts to do direct measurements of these propagators were not informative due to statistical noise. In the future we hope to address these crucial questions using improved wavefunction techniques borrowed from lattice QCD spectrum calculations.

\section{Acknowledgement}

The computer simulation data presented and analyzed here required 3000 hours on one $8 \mathrm{~K}$ quad of the Connection Machine (CM-2) of NCSA. Simulations were also done using the CM-2 at NPAC and PSC, while the ST-100 of Argonne National Laboratory contributed some data before its untimely demise. The work of J. B. Kogut is supported in part by the National Science Foundation, NSF-PHY97-00148. D. K. Sinclair is supported by DOE contract W-31-109-ENG-38. 


\section{References}

1. E. Dagotto and J. B. Kogut, Phys. Rev. Lett. 59, 617 (1987).

2. E. Dagotto, A. Kocić and J. B. Kogut, Phys. Rev. Lett. 60, 772 (1988); 61, 2416 (1988).

3. S. J. Hands, R. L. Renken, A. Kocić, J. B. Kogut, D. K. Sinclair and K. C. Wang, Phys. Lett. B261, 294 (1991).

4. L. D. Landau and I. Ya. Pomeranchuk, Dokl. Akad. Nauk 102, 489 (1955).

5. T. DeGrand and D. Toussaint, Phys. Rev. D22, 2478 (1980); J. Cardy, Nucl. Phys. B170[FS1], 369 (1980); A. Ukawa, P. windey and A. Guth, Phys. Rev. D21, 1013 (1980).

6. P.A.M. Dirac, Phys. Rev. 74, 817 (1948).

7. W. Bardeen, T. Leung and S. Love, Nucl. Phys. B273, 649 (1986); W. Bardeen, S. Love and V. Miransky, Phys. Rev. D42, 3514 (1990).

8. A. Kocić, S. Hands, E. Dagotto and J. B. Kogut, Nucl. Phys. B347, 217 (1990).

9. E. Dagotto and J. B. Kogut, Nucl. Phys. B295[FS21], 123 (1988); E. Dagotto, A. Kocić and J. B. Kogut, ibid. B317, 271 (1989); B331, 500 (1990).

10. S. Duane, Nucl. Phys. B257[FS14], 612 (1985).

11. C. Itzykson and J.-M. Drouffe, Statistical Field Theory, Cambridge University Press, Cambridge (1989).

12. S. Hands, E. Dagotto and J.B. Kogut, Nucl. Phys. B333, 551 (1990);

13. S. Hands and R. Wensley, Phys. Rev. Lett. 63, 2169 (1989).

14. S. Hands, A. Kocić and J. B. Kogut, ILL-(TH)-92-6 (to apper in Phys. Lett.).

15. V. Grosch, K. Jansen, J. Jersák, C. Lang, T. Neuhaus and C. Rebbi, Phys. Lett. B162, 171 (1985).

16. S. Kirkpatrick, Phys. Rev. Lett. 36, 69 (1976).

17. S. Gottlieb, W. Liu, R. L. Renken, R. Sugar and D. Toussaint, Phys. Rev. D38, 2245 (1988).

18. A. Kocić, J. B. Kogut and M.-P. Lombardo, " Universal properties of Chiral Symmetry Breaking" Illinois - CERN preprint; A. Kocić, Phys. Lett. B281, 309 (1992).

19. V. Azcoiti, G. DiCarlo and A. F. Grillo, DFTUZ.91/34 and references therein. 


\section{Table 1}

Average plaquette data at various bare fermion masses $m$ and couplings $\beta=1 / e^{2}$ on a $16^{4}$ lattice.

\begin{tabular}{|c|c|c|c|c|}
\hline$\beta$ & $m=.005$ & $m=.010$ & $m=.020$ & $m=.030$ \\
\hline .30 & - & - & $0.7803(2)$ & $0.7815(2)$ \\
\hline .28 & - & - & $0.8332(2)$ & $0.8338(2)$ \\
\hline .27 & - & - & $0.8621(3)$ & $0.8625(3)$ \\
\hline .26 & - & $0.8922(3)$ & $0.8943(2)$ & $0.8949(3)$ \\
\hline .25 & - & $0.9264(4)$ & $0.9283(2)$ & $0.9296(3)$ \\
\hline .24 & - & $0.9631(4)$ & $0.9651(3)$ & $0.9662(3)$ \\
\hline .235 & - & - & $0.9858(3)$ & $0.9869(3)$ \\
\hline .23 & $1.0025(2)$ & $1.0043(4)$ & $1.0070(3)$ & $1.0076(2)$ \\
\hline .225 & $1.0230(4)$ & $1.0266(4)$ & $1.0290(3)$ & $1.0302(3)$ \\
\hline .22 & $1.0465(3)$ & $1.0489(4)$ & $1.0521(3)$ & $1.0539(3)$ \\
\hline .215 & - & - & $1.0772(4)$ & $1.0783(3)$ \\
\hline .21 & - & $1.1011(4)$ & $1.1049(5)$ & $1.1047(3)$ \\
\hline .20 & - & $1.1607(9)$ & $1.1635(5)$ & $1.1628(4)$ \\
\hline .19 & - & - & $1.2300(5)$ & $1.2276(4)$ \\
\hline .18 & - & - & $1.3028(7)$ & $1.2988(4)$ \\
\hline .17 & - & - & $1.3883(6)$ & $1.3818(5)$ \\
\hline .16 & - & - & $1.4821(7)$ & $1.4757(4)$ \\
\hline .15 & - & - & $1.5873(9)$ & $1.5812(4)$ \\
\hline
\end{tabular}




\section{Table 2}

$<\bar{\psi} \psi>$ data at various bare fermion masses $m$ and couplings $\beta=1 / e^{2}$ on a $16^{4}$ lattice.

\begin{tabular}{|c|c|c|c|c|}
\hline$\beta$ & $m=.005$ & $m=.010$ & $m=.020$ & $m=.030$ \\
\hline .30 & - & - & $.0567(1)$ & $.0821(1)$ \\
\hline .28 & - & - & $.0697(1)$ & $.0988(1)$ \\
\hline .27 & - & - & $.0793(2)$ & $.1111(2)$ \\
\hline .26 & - & $.0499(2)$ & $.0924(2)$ & $.1265(2)$ \\
\hline .25 & - & $.0630(4)$ & $.1098(3)$ & $.1452(3)$ \\
\hline .24 & - & $.0824(5)$ & $.1331(3)$ & $.1675(3)$ \\
\hline .235 & - & - & $.1483(4)$ & $.1812(2)$ \\
\hline .23 & $.0764(9)$ & $.1135(8)$ & $.1636(6)$ & $.1955(2)$ \\
\hline .225 & $.0943(8)$ & $.1340(7)$ & $.1809(5)$ & $.2119(3)$ \\
\hline .22 & $.1225(9)$ & $.1539(6)$ & $.1980(6)$ & $.2288(4)$ \\
\hline .215 & - & - & $.2200(11)$ & $.2462(2)$ \\
\hline .21 & - & $.2056(9)$ & $.2395(9)$ & $.2638(3)$ \\
\hline .20 & - & $.2585(14)$ & $.2827(8)$ & $.3019(4)$ \\
\hline .19 & - & - & $.3261(7)$ & $.3409(4)$ \\
\hline .18 & - & - & $.3678(11)$ & $.3772(3)$ \\
\hline .17 & - & - & $.4085(10)$ & $.4138(3)$ \\
\hline .16 & - & - & $.4427(7)$ & $.4481(2)$ \\
\hline .15 & - & - & $.4774(9)$ & $.4792(2)$ \\
\hline
\end{tabular}




\section{Table 3}

Peak of the monopole susceptibility and the order parameter $M=n_{\max } / n_{\text {tot }}$ at $\beta_{c}=.225$ on $L^{4}$ lattices with $L$ ranging from 10 to 18 .

$\begin{array}{ccc}L & \chi_{\max } & M \\ 10 & 53.3(2.4) & .222(12) \\ 12 & 78.3(4.1) & .212(13) \\ 14 & 108.7(6.2) & .193(14) \\ 16 & 143.1(5.2) & .177(7) \\ 18 & 190.4(8.8) & .1630(53)\end{array}$




\section{Table 4}

$m_{\pi}^{2}$ values for various couplings $\beta$ and bare fermion masses $m$.

$\begin{array}{ccccccccc}m \backslash \beta & .20 & .21 & .22 & .225 & .23 & .24 & .25 & .26 \\ .03 & 0.229(3) & 0.246(3) & 0.266(3) & 0.264(12) & 0.279(14) & 0.272(45) & 0.287(27) & 0.293(25) \\ .02 & 0.159(2) & 0.176(2) & 0.197(2) & 0.212(4) & 0.207(4) & 0.237(5) & 0.254(5) & 0.296(6) \\ .01 & 0.083(2) & 0.096(3) & 0.114(4) & 0.124(3) & 0.137(3) & 0.171(9) & 0.214(5) & 0.250(5) \\ .005 & - & - & 0.069(3) & 0.082(2) & 0.095(2) & - & - & -\end{array}$

Table 5

$m_{\sigma}^{2}$ values for various couplings $\beta$ and bare fermion masses $m$.

$\begin{array}{ccccccccc}m \backslash \beta & .20 & .21 & .22 & .225 & .23 & .24 & .25 & .26 \\ .03 & 1.051(49) & 0.992(32) & 0.709(22) & 0.681(16) & 0.662(11) & 0.567(17) & 0.505(10) & 0.522(8) \\ .02 & 1.136(78) & 0.716(48) & 0.570(16) & 0.520(20) & 0.455(13) & 0.452(15) & 0.380(8) & 0.386(7) \\ .01 & 0.610(87) & 0.475(22) & 0.358(12) & 0.339(11) & 0.286(9) & 0.246(7) & 0.252(5) & 0.273(5) \\ .005 & - & - & 0.233(11) & 0.205(6) & 0.173(6) & - & - & -\end{array}$




\section{Table 6}

$m_{\rho}^{2}$ values for various couplings $\beta$ and bare fermion masses $m$.

$\begin{array}{ccccccccc}m \backslash \beta & .20 & .21 & .22 & .225 & .23 & .24 & .25 & .26 \\ .03 & 2.17(15) & 1.073(93) & 0.412(54) & 1.36(11) & 1.24(11) & 1.195(71) & 0.789(34) & 0.837(35) \\ .02 & 0.83(32) & 1.25(91) & - & 1.17(40) & - & - & 0.645(52) & 0.564(99) \\ .01 & 1.15(25) & 1.11(18) & 1.03(17) & 0.956(88) & 0.817(75) & 0.776(62) & 0.767(55) & 0.663(33) \\ .005 & - & - & 0.56(19) & 0.98(32) & 0.57(11) & - & - & -\end{array}$

\section{Table 7}

$m_{A}^{2}$ values for various couplings $\beta$ and bare fermion masses $m$.

$\begin{array}{ccccccccc}m \backslash \beta & .20 & .21 & .22 & .225 & .23 & .24 & .25 & .26 \\ .03 & 2.19(1.23) & - & 1.19(23) & 1.69(38) & 1.61(29) & 1.49(23) & 1.113(97) & 1.201(85) \\ .02 & 1.80(1.23) & 1.99(80) & 1.46(28) & 1.70(21) & 1.85(19) & 1.43(15) & 1.580(97) & 1.160(72) \\ .01 & 0.87(48) & 0.74(28) & 1.39(35) & 1.08(15) & 1.18(14) & 0.920(94) & 0.808(62) & 0.693(35) \\ .005 & - & - & - & - & - & - & - & -\end{array}$




\section{Figure Captions}

1. $\left\langle\bar{\psi} \psi>\right.$ vs. $\beta$ for $m=0.03,0.02,0.01$ and 0.005 for $16^{4}$ lattice. The data is given in Table 1 . The size of the symbols in the figure includes the statistical error bars.

2. $-\ln \langle\bar{\psi} \psi>$ vs. $-\ln m$ plots for $\beta=0.22,0.225$ and 0.23 .

3. $-1 / \ln (m)$ vs. $-1 / \ln \langle\bar{\psi} \psi>$ plots for various $\beta$ values.

4. Equation of State Eq.(2.1) for $\beta_{c}=0.220, \delta=2.75, \beta_{\text {mag }}=0.571$.

5. Equation of State Eq.(2.1) for $\beta_{c}=0.225, \delta=2.31, \beta_{\text {mag }}=0.763$.

6. Equation of State Eq.(2.1) for $\beta_{c}=0.230, \delta=1.89, \beta_{\text {mag }}=1.124$.

7. Monopole susceptibility $\chi$ vs. $\beta$ on a $16^{4}$ lattice and bare fermion masses $0.03,0.02$ and 0.01 .

8. $\ln \chi_{\max }$ vs. $\ln L$ plotted from the data in Table 2 . The lattices range in linear dimension $L=10,12,14$, 16 , and 18 , and the coupling is $\beta_{c}=0.225$.

9. a. Histogram of the monopole order parameter at $\beta=0.24$.

b. Histogram of the monopole order parameter at $\beta=0.23$.

c. Histogram of the monopole order parameter at $\beta=0.225$.

d. Histogram of the monopole order parameter at $\beta=0.22$.

e. Histogram of the monopole order parameter at $\beta=0.21$.

f. Histogram of the monopole order parameter at $\beta=0.20$.

10. $\ln M\left(\beta_{c}, L\right)$ vs. $\ln L$ from the data in Table 3 at $\beta_{c}=0.225$.

11. The monopole concentration $\left\langle\ell>/ N_{\ell}\right.$ (triangles) and the percolation order parameter $M$ (circles) on a $16^{4}$ lattice at $m=0.01$.

12. A typical pion propagator and its fit, $\beta=0.225$ and $m=0.01$.

13. A typical sigma propagator and its fit, $\beta=0.225$ and $m=0.01$.

14. A typical rho propagator and its fit, $\beta=0.225$ and $m=0.01$.

15. A typical $A_{1}$ propagator and its fit, $\beta=0.225$ and $m=0.01$.

16. $M_{\pi}^{2}$ vs. $\beta$ at $m \rightarrow 0$. The plot was obtained by extrapolating the data in Table 4 to $m=0$ as discussed in the text. 
17. $R(m, \beta)$ vs. $m$ for various couplings $\beta 0.200$ (circles), 0.210 (triangles), 0.220 (inverted triangles), 0.225 (hexagons), 0.230 (dark circles), 0.240 (dark squares) and 0.250 (dark triangles).

18. The chiral Equation of State plotted in the form Eq.(5.3) with $\beta_{c}=0.225, \delta=2.31$ and $\beta_{m a g}=0.763$. The line is the best linear fit to the universal function $f$.

19. $R(m, \beta)$ vs. $m$ from Eq.(5.4a) and (5.6) at $\beta$ values coinciding with Fig.17.

20. $M_{\pi}^{2}$ vs. $\left\langle\bar{\psi} \psi>^{2}\right.$ for the data of Tables 2 and 4 .

21. $M_{\pi}^{2} / M_{\sigma}^{2}$ and $M_{\pi}^{2} / M_{\rho}^{2}$ vs. $\beta$ for fixed $m=0.01$.

22. $M_{\rho}^{2} / M_{A_{1}}^{2}$ vs. $\beta$ for $m=0.01$.

23. $M_{\sigma}^{2} / M_{\rho}^{2}$ vs. $\beta$ for $m=0.01$.

24. $M_{\sigma}^{2} / M_{\rho}^{2}$ vs. $M_{\pi}^{2} / M_{\rho}^{2}$ for $m=0.01$. 